\title{
HEEGAARD SPLITTINGS OF THE PAIR OF THE SOLID TORUS AND THE CORE LOOP
}

\author{
Chuichiro HAYASHI* and Koya SHIMOKAWA ${ }^{\dagger}$
}

\begin{abstract}
We show that any Heegaard splitting of the pair of the solid torus $\left(\cong D^{2} \times S^{1}\right)$ and its core loop (an interior point $\left.\times S^{1}\right)$ is standard, using the notion of Heegaard splittings of pairs of 3manifolds and properly imbedded graphs, which is defined in this paper.
\end{abstract}

\section{Introduction}

The bridge presentation of a link in the 3 -sphere $S^{3}$ is introduced by $\mathrm{H}$. Schubert [S] and generalized by H. Doll[D] for a link in a closed orientable 3-manifold. In [HS1], [HS2], [HS3] we generalize it for a 1-submanifold properly imbedded in a compact connected orientable 3-manifold possibly with boundary. Here we recall it.

Let $I=[0,1]$ an interval, $F$ a disjoint union of closed orientable surfaces. A compression body $C$ is a connected orientable 3-manifold obtained from a 3-manifold $W$ homeomorphic to a ball $B$ or $F \times I$ by attaching 0 or several 1-handles on $\partial B$ or $F \times\{1\}$. Set $\partial_{-} C=\emptyset$ when $W \cong B$, and $\partial_{-} C=F \times\{0\}$ when $W \cong F \times I$. Let $\partial_{+} C=\partial C-\partial_{-} C$. In usual definitions $\partial_{-} C$ has no 2-sphere component, but in this paper $\partial_{-} C$ may have 2 -sphere components. A compression body $C$ is called a handlebody if $W \cong B$ and $\partial_{-} C=\emptyset$.

A set of $\operatorname{arcs}\left\{t_{1}, \cdots, t_{n}\right\}$ properly imbedded in a compression body $C$ is trivial if there is a homeomorphism $C \cong Y \cup V$ (where $Y$ is a ball or

${ }^{*}$ The first author was partially supported by Grant-in-Aid for Scientific Research (No. 10740039), Ministry of Education, Science and Culture.

${ }^{\dagger}$ The second author was partially supported by Fellowship of the Japan Society for the Promotion of Science for Japanese Junior Scientists.

2000 Mathematics Subject Classification: 57M25, 57N10.

Servicio de Publicaciones. Universidad Complutense. Madrid, 2001 
homeomorphic to $\partial_{-} C \times I$ and $V$ is a disjoint union of 1-handles) such that each arc $t_{i}$ satisfies one of the following conditions.

(1) $t_{i}$ is vertical, i.e., $t_{i}=$ (a point) $\times I \subset \partial_{-} C \times I$ and $t_{i} \cap V=\emptyset$.

(2) $t_{i}$ is $\partial_{+}$-parallel, i.e., there is an imbedded disc $D \subset C$ such that $\left(\cup_{j} t_{j}\right) \cap D=t_{i} \subset \partial D$ and $D \cap \partial C=\operatorname{cl}\left(\partial D-t_{i}\right) \subset \partial_{+} C$.

We call such a disc $D$ as in the condition (2) a cancelling disc of $t_{i}$. A standard cut and paste argument allows us to take the cancelling discs to be disjoint.

It is well known that every compact connected orientable 3-manifold $M$ has a Heegaard splitting $H$, i.e., $M=C_{1} \cup_{H} C_{2}$, where $C_{1}$ and $C_{2}$ are compression bodies and $H=\partial_{+} C_{1}=\partial_{+} C_{2}$. Let $T$ be a properly imbedded 1-manifold in $M$. The Heegaard splitting $H$ of $M$ is a Heegaard splitting of $(M, T)$ if $H$ is transverse to $T$ and $T_{i}=T \cap C_{i}$ is trivial in $C_{i}$ for $i=1$ and 2. Every pair of a compact connected orientable 3manifold and a properly imbedded 1-manifold has a Heegaard splitting as shown in [Lemma 2.1, HS1]. See also Lemma 2.1 in this paper.

A Heegaard splitting $(M, T)=\left(C_{1}, T_{1}\right) \cup_{H}\left(C_{2}, T_{2}\right)$ is said to be weakly $T$-reducible if there is a disc $D_{i}$ properly imbedded in $C_{i}$ for $i=1$ and 2 such that $D_{i} \cap T=\emptyset, \partial D_{i}$ is an essential loop in $H-T$ and $\partial D_{1} \cap \partial D_{2}=\emptyset$ in $H$. Otherwise the splitting $H$ is strongly $T$ irreducible. The splitting $H$ is $T$-reducible if we can take discs as above so that $\partial D_{1}=\partial D_{2}$ instead of the condition $\partial D_{1} \cap \partial D_{2}=\emptyset$. Otherwise $H$ is $T$-irreducible. If $H$ is $T$-reducible, then we can see that $H$ is weakly $T$-reducible by slightly isotoping $D_{2}$ near $\partial D_{2}$ to be disjoint from $\partial D_{1}$. A Heegaard splitting $(M, T)=\left(C_{1}, T_{1}\right) \cup_{H}\left(C_{2}, T_{2}\right)$ is said to be cancellable if there is a cancelling disc $D_{i}$ of an arc component $t_{i}$ of $T_{i}$ for $i=1$ and 2 such that $\emptyset \neq\left(\partial D_{1} \cap \partial D_{2}\right) \subset(T \cap H)$. We call these discs a cancelling pair of discs. When $H$ is cancellable and $\partial D_{1} \cap \partial D_{2}$ is a single point, isotoping $t_{i}$ along the $\operatorname{disc} D_{i}$ for $i=1$ and 2, we can obtain another Heegaard splitting $H^{\prime}$ of $(M, T)$ such that $\left|T \cap H^{\prime}\right|=|T \cap H|-2$.

A Heegaard splitting $(M, T)=\left(C_{1}, T_{1}\right) \cup_{H}\left(C_{2}, T_{2}\right)$ is said to be stabilized if there is a properly imbedded disc $D_{i}$ disjoint from $T_{i}$ in $C_{i}$ for $i=1$ and 2 such that $\partial D_{1}$ and $\partial D_{2}$ intersect transversely at a single point in $H$.

Here we give a characterization of Heegaard splittings of a pair of solid torus and its core loop. The following theorem is used in the proof 
of [Theorem 1.2, HS1] to extend a result by A.J. Casson and C.McA. Gordon ([Theorem 3.1, CG]) on irreducible and weakly reducible Heegaard splittings and closed incompressible surfaces. There we use this theorem to show that the obtained closed incompressible surface does not bound a solid torus intersecting the 1-submanifold in its core loop.

Theorem 1.1 Let $V$ be a solid torus, $T_{0}$ a core loop of $V$, and $H$ a Heegaard splitting of $\left(V, T_{0}\right)$. Then $H$ is either stabilized or cancellable. Moreover, when $\left|H \cap T_{0}\right|=2$ and genus $(H) \geq 2, H$ is stabilized.

Heegaard splittings of handlebodies can be easily classified. See [Lemma 2.7, ST].

\section{Heegaard splittings of imbedded graphs in 3- manifolds}

H. Goda defined bridge presentations for theta curves in [G]. Here we generalize it for pairs of compact connected orientable 3-manifolds and 'properly imbedded' graphs.

Let $M$ be a compact 3-manifold possibly with non-empty boundary, and $T$ a (possibly disconnected) graph imbedded in $M$. The graph $T$ is said to be properly imbedded in $M$ if $T \cap \partial M$ consists of all the valency one vertices of $T$. Let $\partial T$ denote the union of these vertices.

Let $C$ be a compression body and $T$ a graph properly imbedded in $C$. The graph $T$ is said to be trivial in $C$ if there is a homeomorphism $C \cong Y \cup V$ as below, where $Y$ is a ball or homeomorphic to $\partial_{-} C \times I$ and $V$ is a disjoint union of 1-handles.

(1) Each component $t$ of $T \cap C$ is an arc or a graph with a single vertex besides $\partial t$,

(2) every arc component of $T \cap C$ is either vertical or $\partial_{+}$-parallel (as in section 1),

(3) for every component $t$ containing a vertex of $T$ besides $\partial t$ there is a properly imbedded disc $D$ such that $D \cap T=t$ and $D \cap \partial C=$ $\partial D \subset \partial C_{+}$.

We can take the disc $D$ in (3) so that it is inessential in $C$, by replacing a disc component $D^{\prime}$ of $D-t$ with a parallel copy of the disc 
$D-D^{\prime}$ slightly isotoped off of $D-\operatorname{cl}\left(D^{\prime}\right)$. By performing this operation on every disc of (3), we can take the discs of (3) to be not nested.

Let $H$ be a Heegaard splitting surface of a compact connected orientable 3-manifold $M$, and $C_{1}, C_{2}$ the compression bodies obtained by cutting $M$ along $H$. Let $T$ be a graph properly imbedded in $M$. We say $H$ is a Heegaard splitting of the pair $(M, T)$ if the vertices of $T$ are disjoint from $H$, the graph $T$ is transverse to $H$ and the graph $T \cap C_{i}$ is trivial in $C_{i}$ for $i=1$ and 2 .

Let $C$ be a compression body, and $T$ a trivial graph properly imbedded in $C$. A spine of $(C, T)$ is a (possibly disconnected when $\partial_{-} C \neq \emptyset$ ) 1-dimensional complex $Q$ (possibly non-properly) imbedded in $C$ such that

(1) $\partial_{+} C \cap Q=\emptyset$,

(2) all the vertical arcs of $T$ are disjoint from $Q$,

(3) every component $t$ of $T$ intersects $Q$ at precisely one vertex of $Q$ if $t$ is not a vertical arc,

(4) for every component $t$ of $T$ with a vertex $p$ besides $\partial t$, the 1complex $Q$ intersects $t$ at the vertex $p$ and

(5) $C$ collapses to $\partial_{-} C \cup Q \cup T$.

When $T=\emptyset$, we call it a spine of $C$.

Lemma 2.1. Let $M$ be a compact connected orientable 3-manifold possibly with boundary, and $H$ be a Heegaard splitting of $M$. Let $T$ be a properly imbedded graph in $M$. Then we can move $T$ by an ambient isotopy of $M$ so that $H$ gives a Heegaard splitting of $(M, T)$.

Proof. The manifold $M$ is decomposed into two compression bodies $C_{1}$ and $C_{2}$, where $H=\partial_{+} C_{1}=\partial_{+} C_{2}$. Let $X_{i}$ be a spine of $C_{i}$ for $i=1$ and 2. We can isotope $T$ to be disjoint from $N\left(X_{1} \cup X_{2}\right)$. Then $M$-int $\left(N\left(X_{1}\right) \cup N\left(X_{2}\right)\right)$ is homeomorphic to $H \times I$ where $I=[0,1]$, $H=H \times\{1 / 2\}$ and $H \times\{0\} \subset C_{1}$. Let $\pi$ be the projection $H \times I \rightarrow$ $H \times\{1 / 2\}$. We can take this product structure so that the singular set of $\pi(T)$ consists of double points away from the vertices of $T$. Let $v$ be a vertex of $T$. Let $S$ be the set consisting of the vertices of $T$ 
and the singular points on $T$, the over ones and the under ones, with respect to the projection map $\pi$. We can isotope $T$ in $M$ so that short arcs containing the over singular points are contained in $C_{2}$ and so that short arcs containing the under singular points are contained in $C_{1}$. (Note that the points of $\partial T$ are also vertices.) We take regular points of $T$, one between every pair of adjacent points of $S$. We take regular points of $T$, one more between adjacent points of $S$ if both points are contained in $C_{1}$ or in $C_{2}$. In addition we take two regular points of $T$ on every circle component of $T$ without singular points. Then we can isotope $T$ so that $T \cap H$ consists of the above regular points and $T \cap C_{i}$ is trivial in $C_{i}$ for $i=1$ and 2 .

In general, let $X$ be a compact 3-manifold, and $T$ a graph properly imbedded in $X$. Let $F$ be a union of compact surfaces in $X$. Suppose that every surface (connected component) $F_{0}$ of $F$ satisfies (1) or (2) below. (1) $F_{0}$ is properly imbedded in $X$ transversely to $T$. (2) $F_{0}$ is imbedded in $X, \partial F_{0} \subset(\partial X \cup T),\left(F_{0} \cap \partial X\right) \subset \partial F_{0}$, and $F_{0} \cap T$ is a union of edges of $T$. In addition, suppose that distinct surfaces of $F$ may intersect each other in a union of edges of $T$. Then we say that $F$ is $T$-compressible if there is a disc $D$ such that $D \cap T=\emptyset, D \cap F=\partial D$ is essential in $F-T$, that is, $\partial D$ does not bound a disc in $F-T$. We call such a disc $D$ a $T$-compressing disc of $F$. If $F$ is not $T$-compressible, then it is $T$-incompressible. We say that $F$ is $T$ - $\partial$-compressible if there is a disc $D$ such that $D \cap T=\emptyset, D \cap(F \cup \partial X)=\partial D$ and $\partial D \cap F=\alpha$ is an essential arc in $F-T$, that is, $\alpha$ does not cobound a disc with a subarc of $\partial F-T$ on $F-T$. We call such a disc $D T$ - $\partial$-compressing disc of $F$. If $F$ is not $T$ - $\partial$-compressible, then it is $T$ - $\partial$-incompressible.

Lemma 2.2. Let $B$ be a ball, and $T$ a connected graph with a single vertex $v$ besides $\partial T$. Suppose that $T$ is properly imbedded in $B$ and trivial in $B$. Let $F$ be a union of compact surfaces in $B$. Suppose for every surface $F_{0}$ of $F$ that $F_{0}$ is imbedded in $B, \partial F_{0} \subset(\partial B \cup T)$, $\left(F_{0} \cap \partial B\right) \subset \partial F_{0}$ and $F_{0} \cap T$ is a union of edges of $T$. In addition, suppose that distinct surfaces of $F$ may intersect each other in a union of edges of $T$. If $F$ is T-incompressible and $T$ - $\partial$-incompressible, then $F$ is a union of

(1) spheres disjoint from $T$,

(2) properly imbedded discs disjoint from $T$, 
(3) discs such that the boundary loop $\ell$ of each of them intersects $\partial B$ in an arc $\alpha$ and such that the arc $\operatorname{cl}(\ell-\alpha)$ is in $T$,

(4) discs which are unions of discs of the above type (3) (see Figure 2.1) and

(5) properly imbedded discs each of which contains a single edge of $T$ with one of the endpoints $v$ in its interior.

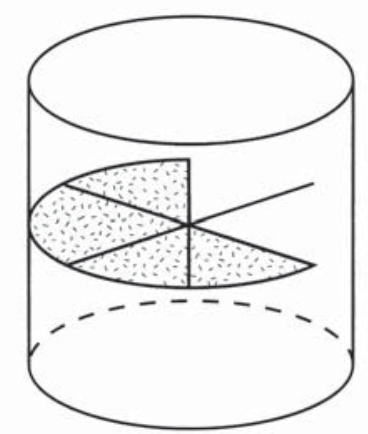

Figure 2.1

Proof. Let $N(T)$ be a small regular neighbourhood of $T$ in $B$. We can take $N(T)$ so that it is a union of a ball neighbourhood $N(v)$ of the vertex $v$ and very small tubular neighbourhoods of the edges of $T$, and each tubular neighbourhood intersects $\partial N(v)$ in a very small disc.

Claim 2.3. Let $\rho$ be an arc which is a union of an $\operatorname{arc} \rho_{0}$ in the punctured sphere $\partial N(T) \cap \partial N(v)$, and two essential arcs in the annuli $\operatorname{cl}(\partial N(T)-(\partial N(v) \cup \partial B))$. Then $\rho$ and an arc in $\partial B$ together form a loop which bounds a disc properly imbedded in $\operatorname{cl}(B-N(T))$.

Proof of Claim 2.3. We can coordinate $B=\left\{(x, y, z) \in \mathbb{R}^{3} \mid x^{2}+y^{2}+\right.$ $\left.z^{2} \leq 1\right\}$ so that $v=(0,0,0)$ and the edges of $T$ are straight line segments connecting $v$ and $\partial B$. Set $B_{\epsilon}=\left\{(x, y, z) \in \mathbb{R}^{3} \mid x^{2}+y^{2}+z^{2} \leq \epsilon^{2}\right\}$ for a small positive real number $\epsilon$. We can take the coordinates so that $N(T)$ is a union of $B_{\epsilon}$ and line segments connecting $v$ and $\partial B$. Let $D$ denote the disc which is the union of straight line segments connecting $v$ and $\partial B$ and intersecting the $\operatorname{arc} \rho_{0}$. Then $D \cap \operatorname{cl}(B-N(T))$ gives a disc as desired. 
Suppose first that $F$ contains a surface which contains an $\operatorname{arc} \lambda \subset T$ properly imbedded in $B$. We take the arc $\lambda$ to be outermost in $F$, that is, there is a regular neighbourhood $Q$ of $v$ in $F$ such that $\lambda \cap Q$ and a subarc of $\partial Q$ cobound a disc $Q^{\prime}$ with $Q^{\prime} \cap T=\lambda \cap Q^{\prime}$. Then $\lambda$ and an arc in $\partial B$ together form a loop bounding a disc $D$ in $B$ such that $D \cap T=\lambda$. By Claim 2.3, we can take $D$ so that $D$ intersects $F$ precisely in $\lambda$ near $\lambda$. Since $F$ is $T$-incompressible and $T$ - $\partial$-incompressible, by a standard innermost loop and outermost arc argument we can see that we can take $D$ so that $D \cap F=\lambda$. We isotope $D$ near $\lambda$ slightly so that $F \cap D$ is an arc $\lambda^{\prime}$ parallel to $\lambda$. Then $\lambda^{\prime}$ and a subarc of $\partial F-T$ together form a loop which bounds a disc $D^{\prime}$ on $F-T$ since $F$ is $T$ - $\partial$-incompressible. Hence the surface of $F$ contains a subdisc of type (3). We repeat the argument as above for every outermost $\operatorname{arc}$ in $T \cap F$, to conclude that the surface of $F$ is a disc of type (3) or (4).

Suppose secondly that $F$ contains a surface which contains an edge $e$ of $T$ as a connected component of $T \cap F$. Then one of the endpoints $v$ of $e$ is in the interior of $F$ and the other endpoint of $e$ is in $\partial F$. Hence similar argument as in the previous paragraph shows that the component is of type (5).

Thus we can assume that $F$ is disjoint from $T$. There is a disc $R$ which is properly imbedded in $B$ and contains $T$. Since $F$ is $T$ incompressible and $T$ - $\partial$-incompressible, we can take $R$ so that it is disjoint from $F$. Then we obtain two balls by cutting $B$ along $R$, and $F$ is properly imbedded in the two balls. Hence $F$ consists of surfaces of types (1) and (2).

Let $C$ be a compression body, and $T$ a trivial graph properly imbedded in $C$. We cap off each sphere component of $\partial_{-} C$ with a 3 -ball if it is disjoint from $T$, to obtain a new compression body denoted by $\tilde{C}$ in the next lemma. Discs and annuli imbedded in $(C, T)$ are called vertical if they are disjoint union of vertical arcs with respect to some homeomorphism $C \cong Y \cup V$ as in the definition for $T$ to be trivial.

Lemma 2.4. Let $C$ be a compression body, and $T$ a trivial graph properly imbedded in $C$. Let $F$ be a union of compact surfaces in $C$. Suppose for every surface $F_{0}$ of $F$ that $F_{0}$ is imbedded in $C, \partial F_{0} \subset(\partial C \cup T)$, $\left(F_{0} \cap \partial C\right) \subset \partial F_{0}$ and $F_{0} \cap T$ is a union of edges of $T$. In addition, suppose that distinct surfaces of $F$ may intersect in a union of edges of $T$. If $F$ is $T$-incompressible and $T$ - $\partial$-incompressible, then $F$ is a union 
CHUICHIRO HAYASHI AND KOYA SHIMOKAWA HEEGAARD SPLITTINGS OF THE PAIR ...

of

(1) spheres disjoint from $T$,

(2) properly imbedded discs disjoint from $T$,

(3) cancelling discs of $\partial_{+}$-parallel arc components of $T$,

(4) properly imbedded discs which are unions of two cancelling discs,

(5) discs such that the boundary loop $\ell$ of each of them intersects $\partial C$ in an arc $\alpha$ and such that the arc $\operatorname{cl}(\ell-\alpha)$ is in $T$,

(6) discs which are unions of discs of the above type (5),

(7) properly imbedded discs each of which intersects $T$ in a single edge of $T$ with an endpoint in its interior,

(8) vertical discs each of which intersects $T$ in two or more vertical arc components,

(9) vertical annuli each of which intersects $T$ in zero or more vertical arc components and

(10) closed surfaces parallel to a component of $\partial_{-} \tilde{C}$ disjoint from $T$.

Proof. Let $t_{1}, \cdots, t_{n}$ be the components of $T$ such that each $t_{i}$ contains a vertex of $T$ besides $\partial t_{i}$. There are discs $D_{1}, \cdots, D_{n}$ properly imbedded in $C$ such that $D_{i}$ contains $t_{i}$ for $i=1, \cdots, n$. We can take these discs so that $D_{i}$ cuts off a ball $B_{i}$ from $C$ with $B_{i} \cap T=D_{i} \cap T=t_{i}$ for $i=1, \cdots, n$. We take parallel copies $D_{1}^{\prime}, \cdots, D_{n}^{\prime}$ of these discs so that $D_{i}^{\prime}$ cuts off a ball $B_{i}^{\prime}$ from $C$ with $B_{i}^{\prime} \cap T=t_{i}$ for $i=1, \cdots, n$. Since $F$ is $T$-incompressible and $T$ - $\partial$-incompressible, by a standard innermost loop and outermost arc argument on $F \cap\left(\cup_{i} D_{i}^{\prime}\right)$, we can deform $F$ to be disjoint from $\cup_{i} D_{i}^{\prime}$ as usual. Note that this operation does not change whether $F$ is a union of surfaces of types (1)-(10) in the statement of this lemma or not.

By Lemma 2.2 the components of $F$ in $B_{i}^{\prime}$ are surfaces of types in the statement of this lemma. By similar argument as in [Lemma 2.1, HS2] the remainder components of $F$ are also surfaces of types in the statement of this lemma. 
The next theorem is a generalization of Haken's theorem [Theorem in section $7, \mathrm{H}]$. The proof of the next theorem is the same as that of [Theorem 1.3, HS1]. We omit the proof. The only difference is that we use the above Lemma 2.4 in this paper instead of Lemma 2.4 in [HS1].

Let $M$ be a compact connected orientable 3-manifold, and $T$ a properly imbedded graph in $M$. The pair $(M, T)$ is split if the complement $M-T$ contains an essential sphere $S$, that is, $S$ does not bound a ball in $M-T$. This sphere $S$ is called a splitting sphere.

Theorem 2.5. Let $H$ be a Heegaard splitting of $(M, T)$, and $S$ a disjoint union of splitting spheres in $(M, T)$ and $T$-compressing discs of $\partial M$. Then there is a disjoint union $S^{\prime}$ of splitting spheres and T-compressing discs such that

(1) $S^{\prime}$ is obtained from $S$ by 2-surgeries and isotopy in $(M, T)$,

(2) each sphere of $S^{\prime}$ intersects $H$ in at most one simple closed curve and

(3) each disc of $S^{\prime}$ intersects $H$ in precisely one simple closed curve essential on $H-T$.

Here a 2-surgery on $S$ is such an operation as taking a disc $D$ with $D \cap S=\partial D$ and $D \cap T=\emptyset$ and replacing a small annular neighbourhood of $\partial D$ in $S$ by two parallel copies of $D$.

\section{Proof of Theorem 1.1, I}

Let $M$ be a compact 3-manifold possibly with boundary, $T$ a graph properly imbedded in $M$, and $H$ a Heegaard splitting surface of $(M, T)$. Let $C_{1}$ and $C_{2}$ be compression bodies obtained by cutting $M$ along $H$, and set $T_{i}=T \cap C_{i}$ for $i=1$ and 2 . The splitting $H$ is said to be cancellable if there is a disc $D_{i}$ imbedded in $C_{i}$ for $i=1$ and 2 such that (1) $t_{i}=D_{i} \cap T_{i} \subset \partial D_{i}$ is an arc properly imbedded in $C_{i}$ for $i=1$ and 2, (2) $\operatorname{cl}\left(\partial D_{i}-t_{i}\right)=D_{i} \cap \partial_{+} C_{i}$ for $i=1$ and 2, (3) $D_{1} \cap D_{2}$ consists of one or two points of $T \cap H$ and (4) either $t_{1}$ and $t_{2}$ are $\partial_{+}$-parallel arc component of $T_{1}$ and $T_{2}$ respectively, or the components of $T_{j}$ which are adjacent to $t_{i}$ are $\partial_{+}$-parallel arc components of $T_{j}$ for $(i, j)=(1,2)$ 
or $(2,1)$. See Figure 3.1. We call the discs $D_{1}$ and $D_{2}$ a cancelling pair of discs. When $D_{1} \cap D_{2}$ consists of precisely one point, we say that $H$ is strongly cancellable. We assume that $H$ is strongly cancellable and $t_{1}$ and $t_{2}$ are $\partial_{+}$-parallel arc components of $T_{1}$ and $T_{2}$ respectively. Then we can obtain another Heegaard splitting of $(M, T)$ by isotoping $t_{2}$ along the disc $D_{2}$ and further isotoping it slightly into $C_{1}$ beyond $H$. Next assume that $H$ is strongly cancellable, that $t_{2}$ contains precisely one vertex $v$ of $T$ with its valency equal to 3 and that the components of $T_{1}$ adjacent to $t_{2}$ are $\partial_{+}$-parallel arcs. Let $e_{1}$ and $e_{2}$ be the edges of $T_{2}$ which together compose $t_{2}$ such that $e_{2}$ is adjacent to $t_{1}=T \cap D_{1}$ on $T$. Then we can obtain another Heegaard splitting of $(M, T)$ by isotoping $e_{2}$ along the disc $D_{2}$ and further isotoping it slightly into $C_{1}$ beyond $H$. In this operation, the vertex $v$ is slid into the compression body $C_{1}$ along the edge $e_{1}$. This can also be pursued by moving $T$ by an ambient isotopy of $M$. We call these two operations cancelling operations.

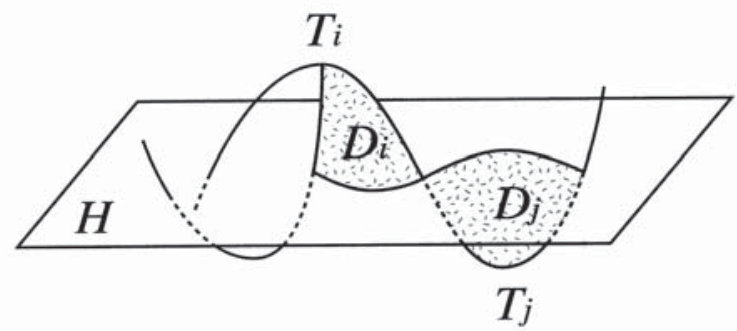

Figure 3.1

Let $M$ be a compact connected orientable 3-manifold, and $T$ a graph properly imbedded in $M$. A Heegaard splitting $(M, T)=\left(C_{1}, T_{1}\right) \cup_{H}$ $\left(C_{2}, T_{2}\right)$ is said to be stabilized if there is a properly imbedded disc $D_{i}$ disjoint from $T_{i}$ in $C_{i}$ for $i=1$ and 2 such that $\partial D_{1}$ and $\partial D_{2}$ intersect transversely at a single point in $H$.

Now we concentrate on our situation. Let $\left(V, T_{0}\right)$ be the pair of the solid torus and the core loop. We take a meridian disc $D$ of $V$ so that $D$ intersects $T_{0}$ transversely in exactly one point $p$. We take an imbedded annulus $A$ such that $T_{0} \subset \partial A, A \cap \partial V=\partial A-T_{0}$ and $A \cap D$ is an $\operatorname{arc} T^{\prime}$ connecting the point $p$ and a point in $\partial V$. Let $T=T_{0} \cup T^{\prime}$ the properly imbedded graph in $V$ with the vertex $p$ of valency 3 and the vertex $\partial T$ 
of valency 1 .

Theorem 3.1 Every Heegaard splitting $H$ of $(V, T)$ is stabilized or cancellable. Moreover, $H$ is stabilized when genus $(H) \geq 2$ and when $H$ is not strongly cancellable. In addition, if two closed surfaces of genus 1 give Heegaard splittings of $(V, T)$ which are not strongly cancellable, then they are isotopic in $(V, T)$.

We will give a proof of Theorem 3.1 later. Theorem 1.1 follows from Theorem 3.1 as below.

Proof of Theorem 1.1 assuming Theorem 3.1. Let $H$ be a Heegaard splitting surface of the pair $\left(V, T_{0}\right)$ of the solid torus and the core loop. Let $C_{1}$ and $C_{2}$ be the compression bodies obtained by cutting $V$ along $H$. Assume that $C_{2}$ contains the boundary torus $\partial V$. We take a point, say $p$, in $T_{0}-H$. We take a meridian disc $D$ of $V$ and an imbedded annulus $A$ so that $D$ intersects $T_{0}$ transversely in the single point $p$ and that $T_{0} \subset \partial A, A \cap \partial V=\partial A-T_{0}$ and $A \cap D$ is an arc, say $T^{\prime}$. Let $T=T_{0} \cup T^{\prime}$ a graph with the vertex $p$ of valency 3 and the vertex $\partial T$ of valency 1 . We will isotope the $\operatorname{arc} T^{\prime}$ so that $H$ gives a Heegaard splitting of $(V, T)$.

Let $X_{i}$ be a spine of $C_{i}$ for $i=1$ and 2 . Then $M-\operatorname{int}\left(N\left(X_{1} \cup X_{2}\right)\right)$ is homeomorphic to $H \times I$ where $I=[0,1], H=H \times\{1 / 2\}$ and $H \times\{0\} \subset$ $C_{1}$. Let $\pi: H \times I \rightarrow H \times\{1 / 2\}$ be the projection.

We can move $X_{i}$ and the $\operatorname{arc} T^{\prime}$ by a small isotopy and take a neighbourhood so that $T \cap N\left(X_{1} \cup X_{2}\right)=\emptyset$ and so that $T$ is transverse to $H$. We can isotope $T^{\prime}$ so that the singular set of $\pi(T)$ consists of double points disjoint from the vertices $p$ and $\partial T$. Suppose there is a double point $x$ of $\pi\left(T^{\prime}\right) \cap \pi\left(T_{0}\right)$ such that the point $\pi^{-1}(x) \cap T_{0}$ is contained in an arc, say $\alpha$, of $T_{0} \cap C_{2}$ and the point $\pi^{-1}(x) \cap T^{\prime}$ is contained in an arc, say $\beta$, of $T^{\prime} \cap C_{2}$, and that $\pi^{-1}(x) \cap \beta$ is over $\pi^{-1}(x) \cap \alpha$ with respect to the second coordinate of the product structure $H \times I$. By retaking the point $x$ if necessary, we assume that $x$ is outermost along $\pi(\alpha)$, that is, there is neither such a double point nor the vertex $p$ between $x$ and an endpoint, say $y$, of $\pi(\alpha)$. Let $\rho$ be the subarc of $\pi(\alpha)$ between $x$ and $y$. We isotope $T^{\prime}$ so that $\pi\left(T^{\prime}\right)$ is isotoped along $\rho$. See Figure 3.2. By repeating this operation, we can eliminate such double points. If there is a double point $z$ of $\pi\left(T^{\prime}\right) \cap \pi\left(T_{0}\right)$ such that the point of $\pi^{-1}(z) \cap T_{0}$ is contained in an arc, say $\gamma$, of $T_{0} \cap C_{1}$ and the point $\pi^{-1}(z) \cap T^{\prime}$ is 
contained in an arc, say $\delta$, of $T^{\prime} \cap C_{1}$, and that $\pi^{-1}(z) \cap \delta$ is under $\pi^{-1}(z) \cap \gamma$, then in the same way we can eliminate $z$. Note that these operations do not yield such a new double point as above. Hence these repetitions terminate in finite times.

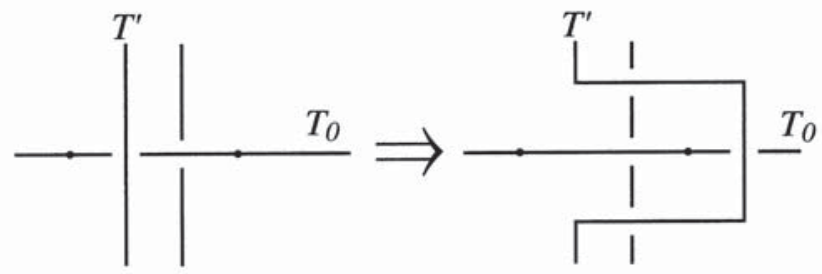

Figure 3.2

Then as in the proof of Lemma 2.1, we let $S$ be the set consisting of the vertices $p, \partial T$ and singular points of $T^{\prime}$ with respect to the projection map $\pi$. We take regular points of $T^{\prime}$, one between every pair of adjacent points of $S$. We take regular points of $T^{\prime}$, one more between every pair of adjacent points of $S$ if both points are Then we isotope $T^{\prime}$ so that $T^{\prime} \cap H$ consists of the above regular points and $T_{i}=T \cap C_{i}$ is trivial in $C_{i}$ for $i=1$ and 2 .

Now, $H$ gives a Heegaard splitting of $(V, T)$. By Theorem 3.1, $H$ is stabilized or cancellable. Moreover, $H$ is stabilized when genus $(H) \geq 2$ and when $H$ is not strongly cancellable.

If $H$ is stabilized as a Heegaard splitting of $(V, T)$, then it is also stabilized as a Heegaard splitting of $\left(V, T_{0}\right)$.

Suppose that the Heegaard splitting $H$ of $(V, T)$ is cancellable. Let $D_{1} \subset C_{1}$ and $D_{2} \subset C_{2}$ be a cancelling pair of discs, and set $t_{i}=D_{i} \cap T$ for $i=1$ and 2 . Then $t_{i}$ is an arc properly imbedded in $C_{i}$. (Note that $\partial C_{i}$ does not go through an edge of $T_{i}$ two or more times by condition (1) of the definition of cancellability.) There are three cases.

(1) $\left(t_{1} \cup t_{2}\right) \subset T^{\prime}$,

(2) $t_{1} \cup t_{2}$ is entirely contained in neither $T_{0}$ nor $T^{\prime}$, or

(3) $\left(t_{1} \cup t_{2}\right) \subset T_{0}$.

In the cases of (1) and (2), since $T^{\prime}$ is an $\operatorname{arc}, D_{1} \cap D_{2}$ is a single point of $T \cap H$. Hence in both cases we can obtain a new Heegaard splitting 
$H^{\prime}$ of $(V, T)$ by a cancelling operation such that $\left|T^{\prime} \cap H^{\prime}\right|=\left|T^{\prime} \cap H\right|-2$ in the case (1) and $\left|T^{\prime} \cap H^{\prime}\right|=\left|T^{\prime} \cap H\right|-1$ in the case (2). (This holds even when $t_{2}$ is an arc component of $T_{0} \cap C_{2}$, and when $t_{1}$ is a union of a subarc of $T_{0} \cap C_{1}$ and a subarc $t_{1}^{\prime}$ of $T^{\prime}$. Because the component of $T^{\prime} \cap C_{2}$ which is adjacent to the subarc $t_{1}^{\prime}$ is a $\partial_{+}$-parallel arc component by condition (4) of the definition of cancellability.) By repeating these operations, we can assume that the cases (1) and (2) never occur.

Hence the case (3) happens. Then it follows that $H$ is also cancellable as a Heegaard splitting of $\left(V, T_{0}\right)$. (This holds even when $t_{1} \cup t_{2}$ contains the vertex $p$ since all we have to do is forgetting the arc $T^{\prime}$.) When $\left|H \cap T_{0}\right|=2$ and genus $(H) \geq 2, H$ is not strongly cancellable as a Heegaard splitting of $(V, T)$. Hence $H$ is stabilized by Theorem 3.1.

We will prove Theorem 3.1 in this and the next sections.

Lemma 3.2. The Heegaard splitting $H$ of $(V, T)$ is stabilized if it is T-reducible.

Proof. If $H$ is $T$-reducible, then there is a sphere $S$ disjoint from $T$ and intersecting $H$ in a single simple closed curve essential in $H-T$. Since $V$ is irreducible, $T$ is connected and $T \cap \partial V \neq \emptyset, S$ bounds a ball disjoint from $T$. Hence $H$ is stabilized by [Satz 3.1, W].

Let $C_{1}, C_{2}$ be the compression bodies obtained by cutting $V$ along the Heegaard splitting surface $H$, and set $T_{i}=T \cap C_{i}$ for $i=1$ and 2. Assume that $C_{2}$ contains the boundary torus $\partial V$. Then $C_{1}$ is a handlebody. We can isotope $D \cup A$ fixing on $T$ so that $D \cup A$ intersects $C_{1}$ in a union of $T_{1}$-incompressible and $T_{1}$ - $\partial$-incompressible surfaces. Moreover, we take $D \cup A$ so that the number $|(D \cup A) \cap H|$ of the intersection loops and arcs is minimal. Then $(D \cup A) \cap C_{1}$ is a union of surfaces of types (2)-(7) in the statement of Lemma 2.4. The discs of type (2) are $T_{1}$-compressing discs of $\partial_{+} C_{1}$ since $|(D \cup A) \cap H|$ is minimal. In addition, a standard cut and paste argument shows that $(D \cup A) \cap C_{2}$ is $T_{2}$-incompressible in $\left(C_{2}, T_{2}\right)$ by the minimality of $|(D \cup A) \cap H|$.

Proposition 3.3. The intersection $(D \cup A) \cap C_{1}$ is a union of surfaces of types (3)-(7) in the statement of Lemma 2.4.

We utilize a method introduced by W. Haken in [section 7, H], and extended by W. Jaco in [Theorem II.7,J], M. Ochiai in [O] and H. Doll in $[\mathrm{D}]$. 
Proof. We assume for a contradiction that $(D \cup A) \cap C_{1}$ contains a disc of type (2). Then $(D \cup A) \cap C_{2}$ contains a $T_{2}$-incompressible nondisc planar surface with a boundary component disjoint from $T$ and in $\partial_{+} C_{2}$, and hence has a $T_{2}$ - $\partial$-compressing disc $D_{1}$ by Lemma 2.4. Set $R_{0}=D \cup A$. We can isotope a small neighbourhood of the arc $\alpha_{1}=D_{1} \cap R_{0}$ along the disc $D_{1}$ into $C_{1}$. Then the resultant union of surfaces in $C_{2}$ are homeomorphic to the surfaces obtained by cutting $R_{0} \cap C_{2}$ along the arc $\alpha_{1}$, and a band is attached to the surfaces $R_{0} \cap C_{1}$ in $C_{1}$. Let $R_{1}$ be the resultant union of surfaces in $V$. Note that $R_{1} \cap C_{2}$ is $T_{2}$-incompressible. Again, it has a $T_{2}$ - $\partial$-compressing disc $D_{2}$ if it does not consist of surfaces of types (2)-(7) and (9). Thus we can perform isotopy along a $T_{2}$ - $\partial$-compressing disc $D_{i}$ of $R_{i-1} \cap C_{2}$ for $i=1, \cdots, n$, until $R_{n} \cap C_{2}$ consists of surfaces of types (2)-(7) and (9). Then the $\operatorname{arcs} \alpha_{i}=D_{i} \cap R_{i-1} \subset R_{0} \cap C_{2}$ divide $R_{0} \cap C_{2}$ into surfaces of types (2)-(7) and (9). We can retake the $T_{2}$ - $\partial$-compressing discs $D_{1}, \cdots, D_{n}$ so that the endpoints of $\alpha_{1}, \cdots, \alpha_{n}$ are in the boundaries of the surfaces $R_{0} \cap C_{1}$. Note that each $\alpha_{i}$ is essential in $\left(R_{0} \cap C_{2}\right)-T_{2}$. The arc $\alpha_{i}$ is called a self-connector if it has distinct endpoints in the same component of $\left(R_{0} \cap C_{1}\right)-T_{1}$. Suppose that every $T_{1}$-compressing disc of $\partial_{+} C_{1}$ in $R_{0} \cap C_{1}$ has a self-connector incident to it. Then there must be an inessential arc $\alpha_{i}$ in $\left(R_{0} \cap C_{2}\right)-T_{2}$ because $R_{0}-T$ is a union of discs. This is a contradiction. Hence there is a $T_{1}$-compressing disc $Q$ among $R_{0} \cap C_{1}$ to which no self-connector is incident. Let $\alpha_{k}$ be the first arc incident to $Q$. The arc $\alpha_{k}$ connects $Q$ to another surface $Q^{\prime}$ of $R_{0} \cap C_{1}$. Then the argument in Lemma 1 in [O] or Lemma 3.3(a) in [D] shows that we can reduce the number $|(D \cup A) \cap H|$ as below.

The $\partial$-compression of $R_{i-1} \cap C_{2}$ along the disc $D_{i}$ can be reversed by a $\partial$-compression of $R_{i} \cap C_{1}$. We call it the dual $\partial$-compression. Let $D_{i}^{\prime}$ denote the dual disc for $D_{i}$, that is, one which gives the dual $\partial$ compression.

First suppose that $D_{k} \cap R_{0}=\alpha_{k}$. Then an isotopy of $R_{0}$ along $D_{k}$ would reduce $|R \cap H|$.

Secondly suppose that $\left(D_{k} \cap R_{0}\right)-\alpha_{k} \neq \emptyset$. Then there are dual $\partial$-compressing discs of $R_{k-1} \cap C_{1}$ intersecting $\beta_{k}=D_{k} \cap H$ among $D_{1}^{\prime}, \cdots, D_{k-1}^{\prime}$. If we isotope $R_{k-1}$ along the discs $D_{k-1}^{\prime}, \cdots, D_{1}^{\prime}$ in this order, then we obtain $R_{0}$. The interior of the discs $D_{1}^{\prime}, \cdots, D_{k-2}^{\prime}$ may intersect $R_{k-1}$. The arc $\beta_{k}$ connects $Q$ and $Q^{\prime}$. Let $D_{j}^{\prime}(j<k)$ be the 
dual disc intersecting $\beta_{k}$ and the nearest to $Q$. Let $E$ be the subdisc of $\partial N\left(\beta_{k}\right) \cap C_{1}$ between $Q$ and $D_{j}^{\prime}$. We retake the dual disc $D_{j}^{\prime}$ so that the subdisc $D_{j}^{\prime} \cap N\left(\beta_{k}\right)$ is replaced by $E \cup\left(Q-N\left(\beta_{k}\right)\right)$, and we isotope the new $D_{j}^{\prime}$ slightly off of $Q$. We perform the above deformation of dual discs for each intersection point of dual discs and $\beta_{k}$, so that dual discs $D_{1}^{\prime}, \cdots, D_{k}^{\prime}$ are disjoint from $\beta_{k}$. Then we can isotope the disc $R_{k-1}$ along the discs $D_{i}^{\prime}(i=k-1, \cdots, 1)$, and further isotope along the disc $D_{k}$, to reduce $|R \cap H|$. This is a contradiction.

\section{Proof of Theorem 1.1, II}

By Proposition 3.3, we can take $R=D \cup A$ so that it intersects $C_{1}$ in surfaces of types (3)-(7) in the statement of Lemma 2.4. Let $Q$ be a spine of $\left(C_{1}, T_{1}\right)$. We can take $Q$ to be a connected 1-complex which intersects $R$ in vertices of $Q$ so that $(Q \cap R) \subset T$. We think of $C_{1}$ as being a very small neighbourhood of $Q$. Let $G$ be the ball obtained by cutting the solid torus $V$ along the union $R$ of the meridian disc $D$ and the annulus $A$. Then $Q_{G}=Q \cap G$ is a graph.

The next lemma is essentially Proposition 2.5 in [ST], and this proof is essentially due to $[\mathrm{F}]$.

Lemma 4.1. We can assume that every component of $Q_{G}$ is a tree. Otherwise, $H$ is stabilized.

Proof. Suppose not. Then $Q_{G}$ contains a (possibly knotted) simple closed curve $c$. Let $e$ be an edge of $Q_{G}$ contained in $c$. We slide an endpoint $q$ of $e$ along the arc $c-e$, to deform $e$ into a loop edge. We slide $q$ slightly on $e$ passing through the other endpoint, and obtain a loop $\ell \subset e$ imbedded in the interior of $G$. We take a small neighbourhood $N(\ell)$ of $\ell$ in $C_{1}$. Then $C_{1}-\operatorname{int} N(\ell)$ is a compression body, and $H$ is a Heegaard splitting of $(V-\operatorname{int} N(\ell), T)$. We push the sphere $\partial G$ slightly into int $G$ and obtain a splitting sphere $S$ of $(V-\operatorname{int} N(\ell), T)$, that is, $S$ does not bound a ball in $V-\operatorname{int} N(\ell)-T$. Then by Theorem 2.5 there is a sphere $S^{\prime}$ which is disjoint from $T$ and intersecting $H$ in a single simple closed curve essential in $H-T$. Then $H$ is $T$-reducible as a splitting of $(V-\operatorname{int} N(\ell), T)$, hence also as a splitting of $(V, T)$. Thus the splitting $H$ is stabilized by Lemma 3.2.

Since $T_{0}$ is a loop, $Q \cap T_{0} \neq \emptyset$. In addition, $Q \cap T^{\prime} \neq \emptyset$ since the arc 
$T^{\prime}$ has an endpoint in $\partial V=\partial_{-} C_{2}$ and the other endpoint at the vertex $p=T_{0} \cap T^{\prime}$. However, it is possible that $Q \cap T^{\prime}=p$. Because $R \cap C_{1}$ is a union of surfaces of types (3)-(7) in the statement of Lemma 2.4, each of the discs obtained by cutting $D$ and $A$ along $T^{\prime}$ intersects $C_{2}$ in a disc. Hence the union of surfaces $R \cap C_{2}$ is $T_{2}$-incompressible. Then $R \cap C_{2}$ is $T_{2^{-}} \partial$-compressible by Lemma 2.4. Note that $A \cap C_{2}$ is $T_{2}$ - $\partial$-compressible even when $Q \cap T=p$. We can isotope $R$ along $T_{2}$ - $\partial$-compressing discs $D_{1}, \cdots, D_{n}$ to deform $R \cap C_{2}$ into a union of surfaces of types (3)-(7) and (9) in the statement of Lemma 2.4. Note that the disc of type (2) cannot appear since $R \cap C_{1}$ does not have the disc component of type (2) and since $D_{1}, \cdots, D_{n}$ are $T_{2}$ - $\partial$-compressing discs. Set $R_{0}=R$, and let $R_{i}$ be the resultant union of surfaces obtained by the $i$-th isotopy along the disc $D_{i}$. Let $\gamma_{i}$ be the arc $R_{i-1} \cap \partial D_{i}$, and $\delta_{i}$ the arc $\partial D_{i} \cap \partial_{+} C_{2}$. We can isotope the discs $D_{1}, \cdots, D_{n}$ so that the endpoints of $\gamma_{1}, \cdots, \gamma_{n}$ are in the boundary of the discs $R_{0} \cap C_{1}$. Let $\Gamma_{i}$ be the graph with the edges $\cup_{j=1}^{i} \gamma_{j}$, regarding the disc components of $R_{0} \cap C_{1}$ as vertices. Let $\tilde{\Gamma}_{i}$ be the intersection of $\Gamma_{i}$ and the sphere $\partial G$. The sphere $\partial G$ contains two copies of $D$, two copies of $A$ cut along $T^{\prime}$, a copy of $T_{0}$ cut at $p$ and four copies of $T^{\prime}$. Note that the graph $\tilde{\Gamma}_{i}$ contains two copies of each edge $\gamma_{j}$. See Figure 4.1.

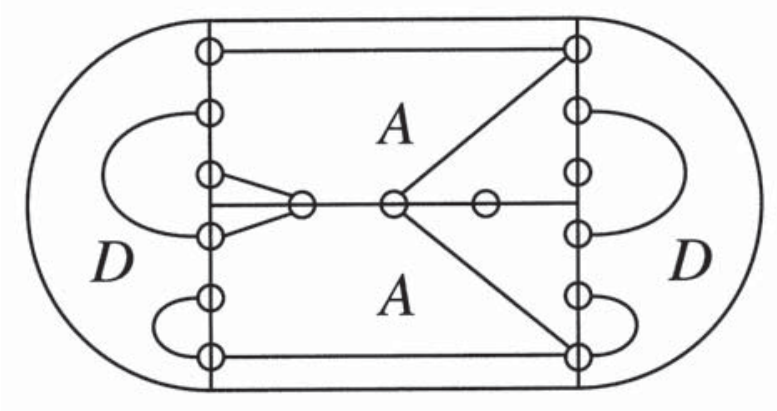

Figure 4.1

We concentrate on the patterns of $\tilde{\Gamma}_{i}$ as below.

(1) An edge $e$ of $\tilde{\Gamma}_{i}$ in $A$.

(2) An edge $e$ of $\tilde{\Gamma}_{i}$ in $D$ such that $e$ connects two points in the same copy of $T^{\prime}$ (Figure 4.2). 
(3) Two edges of $\tilde{\Gamma}_{i}$ in a copy of $D$ such that they connects distinct copies of $T^{\prime}$ and such that they have an endpoint at the same point (Figure 4.3).

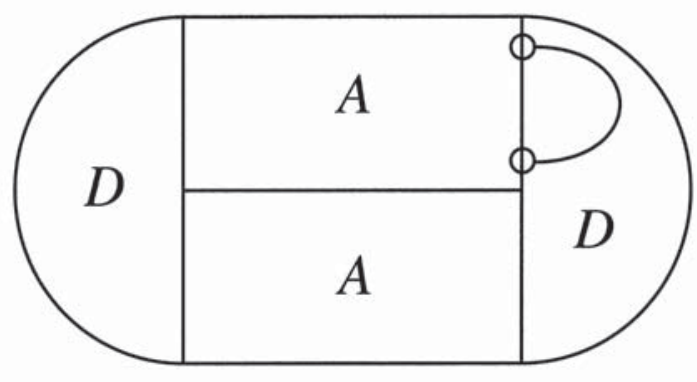

Figure 4.2

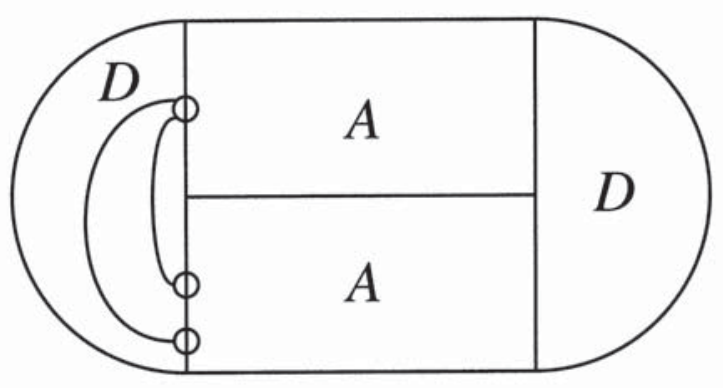

Figure 4.3

We allow the edge $e$ of the above types (1) and (2) to be incident to the vertex $p=T_{0} \cap T^{\prime}$. Let $k$ be the smallest number such that $\tilde{\Gamma}_{k}$ contains such a pattern. Note $i<k$, that $\tilde{\Gamma}_{i}$ is a disjoint union of arcs for $i<k$.

We perform the $i$ th isotopy of $R_{i-1}$ along the $T_{2}$ - $\partial$-compressing disc $D_{i}$ for $i \leq k$ by sliding edges of the spine $Q$ as below. (The description of deformation is completed right after Lemma 4.3.) Let $Q_{i}$ denote the spine after the $i$ th deformation. The original spine is denoted by $Q_{0}$. We will deform the spine so that $Q_{i} \cap R_{i}=\Gamma_{i}$. That is, we allow $i$ edges of $Q_{i}$ to be contained in $R_{i}$. Let $Q_{G i}$ denote the graph $Q_{i} \cap G$. The edges $Q_{i} \cap R_{i}=\Gamma_{i}$ appear twice in the graph $Q_{G i}$. 
Lemma 4.2. We can assume that every component of $Q_{G i}$ is a tree for $i<k$. Otherwise, $H$ is stabilized.

Proof. The proof is similar to that of Lemma 4.1, and we omit the detail. Suppose that $Q_{G i}$ contains a cycle $c$. Since $\tilde{\Gamma}_{i}$ does not contain a cycle, the cycle $c$ contains an edge $e$ such that $e$ is not contained in $\tilde{\Gamma}_{i}$. We slide the edge $e$ as in the proof of Lemma 4.1, and the same argument shows that $H$ is stabilized.

We can assume that the condition of Lemma 4.2 are kept after any sliding operations on edges of $Q_{G i}-\tilde{\Gamma}_{i}$ in $G$. Otherwise, $H$ is stabilized.

We can extend the $T_{2}$ - $\partial$-compressing disc $D_{i}$ so that $\delta_{i} \subset Q_{i-1}$, and isotope $D_{i}$ so that $\delta_{i}$ is a sequence of edges of $Q_{G(i-1)}$, where the same edge possibly appears more than once.

Lemma 4.3. We can deform $Q_{i-1}$ by sliding edges of $Q_{G(i-1)}-\tilde{\Gamma}_{i-1}$ and isotope $D_{i}$ so that $\delta_{i}$ does not intersect an edge of $Q_{G(i-1)}$ more than once.

Proof. Let $x$ and $y$ be points of $\delta_{i}$ which are in the same vertex $v$ of $Q_{G(i-1)}$. We can choose $x$ and $y$ so that the subarc $\rho$ connecting $x$ and $y$ in $\delta_{i}$ contains no such pair of points. Then by Lemma $4.2 \rho$ consists of two successive subarcs of $\delta_{i}$ contained in the same edge of $Q_{G(i-1)}$. See Figure 4.4. Now, we can easily slide edges of $Q_{G(i-1)}-\tilde{\Gamma}_{i-1}$ and isotope $D_{i}$ to shrink $\rho$ into a point at the vertex $v$.

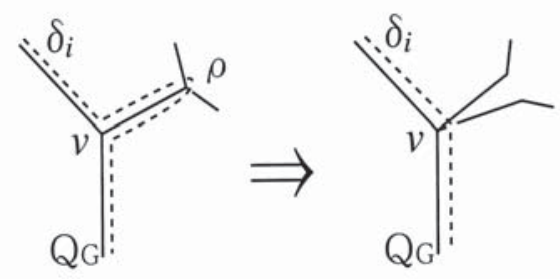

Figure 4.4

Then for $1 \leq i \leq k$ we perform the $i$ th deformation of the spine $Q_{i-1}$ along $D_{i}$ as below. Note that $\partial D_{i}$ cannot entirely be contained in $\partial G$ since every connected component of $\tilde{\Gamma}_{i}$ is an edge in a copy of $D$ such that it connects distinct copies of $T^{\prime}$. Hence $\delta_{i}$ contains an edge $e$ of $Q_{G(i-1)}-\tilde{\Gamma}_{i-1}$, we slide the edge $e$ along the arcs $\delta_{i}-e$ and isotope along the disc $D_{i}$, to push $e$ into $\partial G$. Then $e$ is in the place of $\gamma_{i}$. 
We consider the case where a copy of $\gamma_{k}$ is of type (3) as in Figure 4.3. In the case of type (2), the proof is similar to that of this case and easier, and we omit it.

When a copy of $\gamma_{k}$ is of type (3), in the graph $\tilde{\Gamma}_{k}$ a copy of the edge $\gamma_{k}$ and a copy of some edge $\gamma_{m}$ have an endpoint in the same vertex $v$ of $Q_{G k}$. We call these copies of edges $\gamma_{m}$ and $\gamma_{k}$ for simplicity.

The other endpoints $v_{k} \subset \partial \gamma_{k}$ and $v_{m} \subset \partial \gamma_{m}$ are in distinct vertices in the same copy $t$ of $T^{\prime}$. Let $t^{\prime}$ be the subarc of $t$ between $v_{k}$ and $v_{m}$. See Figure 4.5.

Lemma 4.4. If $\gamma_{k}$ is of type (3), then $H$ is strongly cancellable.

Proof. Let $E$ be the disc bounded by $t^{\prime} \cup \gamma_{k} \cup \gamma_{m}$ in $R_{k}$ right after the $k$ th deformation. Note that $E$ is disjoint from the other edges of $\tilde{\Gamma}_{k}$ than $\gamma_{m}$ and $\gamma_{k}$ because of the definition of the number $k$.

First, suppose that $t^{\prime}$ contains no other vertices than $v_{k}$ and $v_{m}$. Then $t^{\prime}$ is a $\partial_{+}$-parallel arc component of $T_{2}$ and $E$ gives a cancelling disc of $t^{\prime}$ in $\left(C_{2}, T_{2}\right)$. The vertex $v_{m}$ does not coincides with the vertex $p$. Hence the ball neighbourhood $N\left(v_{m}\right)$ contains a cancelling disc $E^{\prime}$ of the $\partial_{+}$-parallel arc of $T_{1}$ corresponding to $v_{m}$. We can take $E^{\prime}$ so that $E^{\prime}$ is perpendicular to the sphere $\partial G$ inside $G$ and so that $E^{\prime}$ is disjoint from the interior of the edges of $Q_{k}$. Then $E$ and $E^{\prime}$ give a cancelling disc pair, and we see that $H$ is strongly cancellable.

Secondly, suppose that $t^{\prime}$ contains other vertices than $v_{k}$ and $v_{m}$. Let $w_{1}, \cdots, w_{r}$ be the vertices contained in $t^{\prime}$ other than $v_{k}$ and $v_{m}$, appearing in this order in $t^{\prime}$ so that $w_{1}$ is the nearest to $v_{k}$. The $\operatorname{arc} t^{\prime}$ is divided into $r+1$ arcs when it is cut at the vertices $w_{1}, \cdots, w_{r}$. Let $t_{i}$ be the subarc of $t^{\prime}$ between $w_{i-1}$ and $w_{i}$, where $v_{k}=w_{0}$ and $v_{m}=w_{r+1}$. The $\operatorname{arc} t_{i}$ is a $\partial_{+}$-parallel arc component of $T_{2}$. Let $E_{i}$ be a cancelling disc of $t_{i}$ in $\left(C_{2}, T_{2}\right)$. We can take $E_{1}, \cdots, E_{r+1}$ so that they are disjoint and they intersect the disc $E$ only properly imbedded arcs connecting vertices of $Q_{k}$. Let $\mathcal{E}=\cup E_{i}$. See Figure 4.5.

If several arcs of $\mathcal{E} \cap E$ are incident to the vertex $v$, then we isotope $\mathcal{E}$ along the edge $\gamma_{k}$ to deform such arcs into arcs incident to the vertex $v_{k}$. See Figures 4.6 and 4.7 .

Suppose there is an $\operatorname{arc} \rho$ of $\mathcal{E} \cap E$ such that $\rho$ has two endpoints in the same vertex $q$. Then we can take $\rho$ to be the outermost one among such $\operatorname{arcs}$ on $E$. That is, there is a disc $E^{\prime}$ cut off by $\rho$ from $E$ such that $E^{\prime}$ is disjoint from such arcs other than $\rho$. We perform a surgery on $\mathcal{E}$ 
CHUICHIRO HAYASHI AND KOYA SHIMOKAWA HEEGAARD SPLITTINGS OF THE PAIR ...

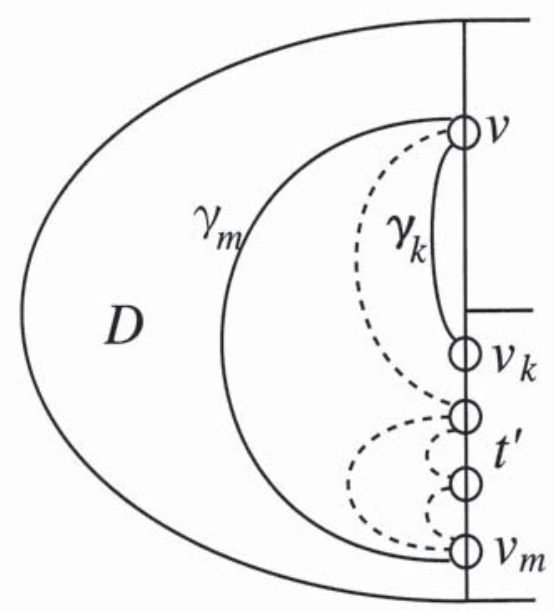

Figure 4.5
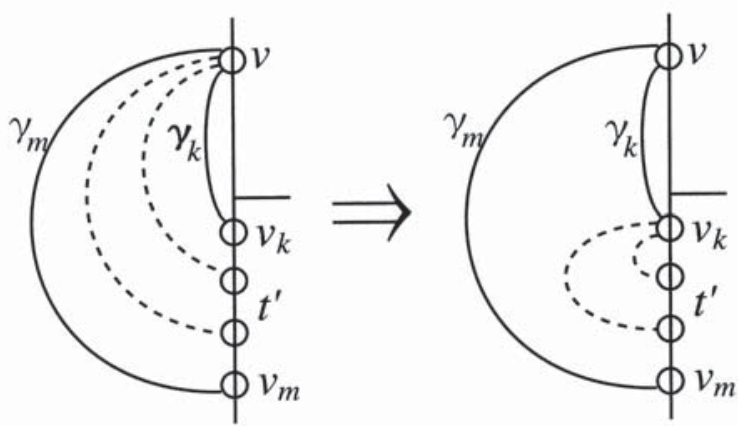

Figure 4.6
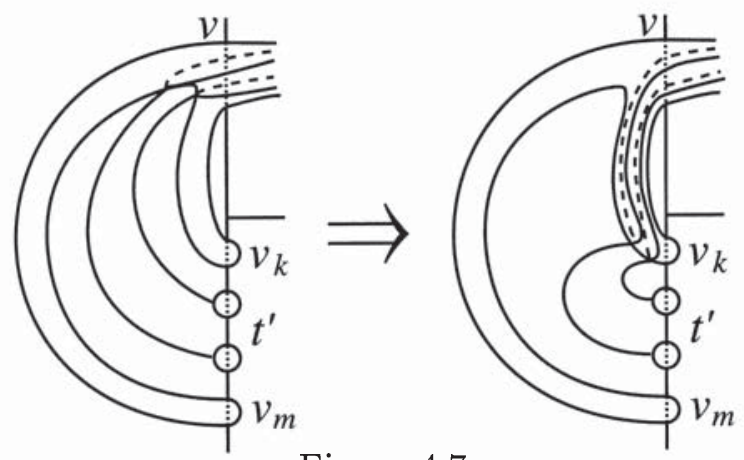

Figure 4.7 
along the disc $E^{\prime}$ and discard the component disjoint from $T_{2}$, to obtain a union of cancelling discs which intersects $E$ in fewer number of arcs. Hence we can assume that $\mathcal{E} \cap E$ does not contain such an arc.

We say that an arc of $\mathcal{E} \cap E$ is good if it connects adjacent vertices. We say a vertex $w_{i}$ is good if all the arcs incident to $w_{i}$ are good. There is such a good vertex $w_{i}$ since every polygon has at least two vertices with no diagonal arc incident when divided into triangles by diagonal arcs. Then all the arcs incident to $w_{i}$ have the other endpoint in the adjacent vertices $w_{i-1}$ and $w_{i+1}$. Hence there is a cancelling disc $E_{i}$ such that (a) $E_{i}$ is a cancelling disc of $t_{i}$ or $t_{i+1}$, (b) $E_{i}$ intersects $E$ transversely in arcs, and (c) only good arcs of $E_{i} \cap E$ are incident to $w_{i}$. Among such discs, we take $E_{i}$ so that the number of intersection $\operatorname{arcs} E_{i} \cap E$ is minimum.

We will show that no arc of $E_{i} \cap E$ is incident to $w_{i}$. Suppose there is an arc $\mu$ of $E_{i} \cap E$ either connecting $w_{i-1}$ and $w_{i}$ or connecting $w_{i}$ and $w_{i+1}$. We consider the former case. The proof of latter case is similar and we omit it. We take $\mu$ to be the outermost one on $E$. The arc $\mu$ divides $E_{i}$ into two discs, one of which, say $E_{i}^{\prime}$ intersects neither $t_{i}$ nor $t_{i+1}$. Let $E^{\prime}$ be the subdisc of $E$ cobounded by $t_{i}$ and $\mu$. Then $E^{\prime} \cup E_{i}^{\prime}$ is a cancelling disc of $t_{i}$ which intersects in fewer number of arcs than $E_{i}$ after an adequate small isotopy. This is a contradiction.

Thus there is no arc of $E_{i} \cap E$ which is incident to $w_{i}$. Let $N\left(w_{i}\right)$ be a ball neighbourhood of the vertex $w_{i}$. The disc $N\left(w_{i}\right) \cap E$ is a cancelling disc of the arc component of $T_{1}$ corresponding to the vertex $w_{i}$. Then the discs $E_{i}$ and $N\left(w_{i}\right) \cap E$ show that $H$ is strongly cancellable.

The proof of the next lemma is similar to that of Lemma 4.4. We omit it.

Lemma 4.5. Suppose that $\gamma_{k}$ is of type (2). Then the Heegaard splitting $H$ is strongly cancellable.

The next lemma completes the proof of the first sentence of Theorem 3.1 .

Lemma 4.6. Suppose that $\gamma_{k}$ is of type (1). Then the Heegaard splitting $H$ is cancellable. Moreover, if $H$ is not strongly cancellable, then $T \cap Q=$ $p$ and $\gamma_{k}$ is an edge connecting the two copies of $p$ in a copy of $A$.

Proof. The $\operatorname{arc} T^{\prime}$ and the edge $\gamma_{k}$ divides the annulus $A$ into two discs, one of which, say $E$, is disjoint from the toral boundary $\partial V$. 
Then similar argument as in the proof of Lemma 4.4 will show that the Heegaard splitting $H$ is cancellable.

When the arc $\partial E-\gamma_{k}$ does not contain a vertex of $Q_{G k}$, then we can take a disc $E^{\prime}$ in $N\left(\partial \gamma_{k}\right)$ so that $E$ and $E^{\prime}$ together form a cancelling pair of discs. In addition, if $\partial \gamma_{k}=p$, then we can take a cancelling disc of the $\operatorname{arc} T_{0} \cap N(p)$ in $N(p)$, and see that $H$ is cancellable. Since we can also take a cancelling disc of the arc $\partial E \cap N(p)$ in $N(p)$, we see that $H$ is strongly cancellable unless $T \cap Q=p$ and $\gamma_{k}$ is an edge connecting the two copies of $p$ in a copy of $A$.

When the arc $\partial E-\gamma_{k}$ contains a vertex of $Q_{G k}$ other than $\partial \gamma_{k}$, the proof is very similar as in that of Lemma 4.4.

The next lemma implies the second and third sentences of the statement of Theorem 3.1.

Lemma 4.7. If $H$ is not stabilized and not strongly cancellable, then genus $(H)=1$. Moreover, such splitting surfaces are isotopic in $(V, T)$.

Proof. Suppose that $H$ is not stabilized and not strongly cancellable. Then by Lemmas 4.4, 4.5 and 4.6 the edge $\gamma_{k}$ is contained in a copy of $A$ and connects two copies of the vertex $p=T_{0} \cap T^{\prime}=T \cap Q_{k}$ as in the statement of Lemma 4.6. Then before the deformation $Q_{G}$ is a connected tree intersecting $\partial G$ in the two copies of the vertex $p$ by Lemma 4.1. We can shrink $Q_{G}$ to be an edge $e$ connecting the two copies of $p$. Thus $Q$ consists of a loop edge incident to $p$, and this implies that genus $(H)=1$. Moreover, the edge $e$ is not knotted in $G$ since $C_{2}$ is homeomorphic to (a torus) $\times I$.

\section{References}

[BO] F. Bonahon and J.-P. Otal, Scindements de Heegaard des espaces lenticulaires, Ann. Sci. Ec. Norm. Sup. 16(4) (1983),451-466.

[CG] A.J. Casson and C.McA. Gordon, Reducing Heegaard splittings, Topology Appl. 27 (1987), 275-283.

[D] H. Doll, A generalized bridge number for links in 3-manifolds, Math. Ann. 294 (1992), 701-717.

[F] C. Frohman, The topological uniqueness of triply periodic minimal surfaces in $\mathbb{R}^{3}$, J. Differ. Geom. 31 (1990), 277-283. 
CHUICHIRO HAYASHI AND KOYA SHIMOKAWA HEEGAARD SPLITTINGS OF THE PAIR ...

[G] H. Goda, Bridge index for theta curves in the 3-sphere, Topology Appl. 79 (1997), 177-196.

[H] W. Haken, Some results on surfaces in 3-manifolds, Studies in Modern Topology (Math. Assoc. Amer., distributed by: Prentice-Hall) (1968), 34-98.

[HS1] C. Hayashi and K. Shimokawa, Thin position for 1-submanifold in 3manifold, Pacific J. Math. 197 (2001), 301-324.

[HS2] C. Hayashi and K. Shimokawa, Heegaard splittings of the trivial knot, J. Knot Ramifi. 7 (1998), 1073-1085.

[HS3] C. Hayashi and K. Shimokawa, Heegaard splittings of trivial arcs in compression bodies, J. Knot Ramifi. 10 (2001), 71-87.

[J] W. Jaco, Lectures on three-manifold topology, (CBMS Reg. Conf. Ser., vol 43). Providence, RI: Am. Math. Soc., 1980.

[O] M. Ochiai, On Haken's Theorem and its extension, Osaka J. Math. 20 (1983), 461-468.

[ST] M. Scharlemann and A. Thompson, Heegaard splitting of (surface) $\times I$ are standard, Math. Ann. 295 (1993), 549-564.

[S] H. Schubert, Ueber eine numerische Knoteninvariante, Math. Z. 61 (1954), 245-288.

[W] F. Waldhausen, Heegaard-Zerlegungen der 3-Sphäre, Topology 7 (1968), 195-203.

Department of Mathematics

Faculty of Science

Gakushuin University

Graduate School of Information Sciences

Tohoku University

1-5-1 Mejiro Toshima-ku Tokyo, 171, Japan

E-mail: Chuichiro.Hayashi@gakushuin.ac.jp

2-1-1 Katahira Aoba-ku Sendai, 980-8577, Japan

E-mail: koya@math.is.tohoku.ac.jp

Recibido: 20 de Marzo de 2000

Revisado: 4 de Diciembre de 2000 